\title{
Convulsive Pseudoseizures: a Review of Current Concepts
}

\author{
GHISLAINE SAVARD and FREDERICK ANDERMANN \\ Department of Neurology and Neurosurgery, McGill University, and The Montreal Neurological \\ Institute and Hospital, Canada
}

Correspondence: Dr. G. Savard, Montreal Neurological Hospital, 3801 University Street, Montreal, Que. H3A 2B4 Canada

\begin{abstract}
Convulsive pseudoseizures thought to represent psychiatric disease can usually be detected early if they are considered in the epileptologist's differential diagnosis. No single diagnostic criterion for this behavioural disorder is known to be pathognomonic. Epilepsy and all physiological explanations have to be thoroughly ruled out and positive evidence of relevant psychopathology has to be gathered.

The presence of pseudoseizures often heralds severe psychopathology, most frequently major affective disorder and major personality disorder, and occasionally, factitious disorder. Pseudoseizures can coexist with epileptic seizures and are often triggered by anticonvulsant toxicity.

Diagnostic problems are more likely to be encountered in patients with frontal or parietal epilepsies, and in patients whose severe psychopathology is refractory to psychiatric intervention.
\end{abstract}

\section{Introduction}

A consensus concerning the diagnostic and therapeutic challenges that pseudoseizures pose is currently emerging from a vast, and growing, literature (Trimble, 1986; Lesser, 1985). It is hoped that agreement on the terminology and the classification of pseudoseizures can also soon be reached, and that the factors predictive of outcome can be identified.

The term pseudoseizure most often refers to non-epileptic seizure-like events unaccompanied by acute cerebral dysfunction nor by paroxysmal epileptiform electrical activity. Usually, pseudoseizures are thought to represent psychiatric disease, especially if a physiological etiology has been thoroughly excluded and the relevant psychopathology demonstrated.

In this review the phenomenology of convulsive pseudoseizures, a subtype widely believed to be the most commonly encountered is discussed. Useful modern investigations are outlined, the psychiatric diagnoses reported in pseudoseizure patients are addressed, and accepted treatment recommendations are alluded to. The goal is to allow for early identification of patients thus afflicted and confident differential diagnostic formulation. This is especially crucial in cases where diagnostic conundrums develop and joint neurological and psychiatric follow-up is required to uncover the etiology and prevent fatalities (Savard and Andermann, 1990). 


\section{Clinical Manifestations}

The onset of a convulsive pseudoseizure has traditionally been described as gradual (Gowers, 1901; Lennox, 1960), but an abrupt beginning is not excluded. A warning of malaise accompanied by the unspecific complaints of dizziness, dyspnea, headache is often voiced to a significant witness whose presence seems to have a permissive influence. Less commonly, micropsia, autoscopic visions, and olfactory and gustatory experiences are mentioned by patients (Lesser, 1985), but these subjective perceptual experiences are rarely correlated with objective behavioural evidence. Nocturnal occurrence is most likely preceded by awakening (Fenton, 1986). An onset during sleep, at times reported by patients, has not been convincingly demonstrated (Green, 1977).

Motor manifestations are varied and include unilateral or bilateral, tonic, clonic, tonic-clonic or vigorous non-purposeful flailing muscle activity, with dystonic posturing frequently associated. Out-of-phase random movements of the upper and lower extremities, pelvic forward thrusting, and forced turning of the head from side to side are particularly characteristic (Gates et al., 1985) while classical opisthotonos, kicking and biting are dramatic but uncommon (Lesser, 1985). Facial expressions attest to a variety of emotions from sadness, rage, ecstasy (Fenton, 1986) as the facial musculature is generally not involved in the generalized jerking.

Vocalizations vary from moans, grunts, screams to vulgar or mystic verbal utterances (Lesser, 1985). Of a quasivolitional quality (Lennox, 1960), they regularly signal the onset of the pseudoseizure (Gates et al., 1985).

Avoidance of pain such as prevention of discomfort during and after the attack is revealing when present; if absent, intentional self-injury may offer the next best explanation if compatible with the individual patient's underlying psychopathology (Lesser, 1985; Fenton, 1986). Urinary and fecal incontinence anecdotally reported by some authors to occur in pseudoseizures should also be understandable in the light of the coexisting psychopathology.

Among autonomic features, cardiorespiratory signs with dilated pupils from increased sympathetic discharge can be the physical concomitants of emotional bewilderment during pseudoseizure (Lesser, 1985). Cyanosis can result from breath-holding (Fenton, 1986).

Like onset, termination may be gradual or abrupt (Lesser, 1985). An interesting physical sign, useful to recognize pseudounconsciousness which may follow an attack, is the geotropic eye movement. This consists of deviation of the eyes towards the ground and away from the examiner, and may be elicited by passive head turning; it appears in distinct contrast to the fixed eye deviation observed during some epileptic seizures (Henry and Woodruff, 1978; Rosenberg, 1982). The presence of a Babinski sign is not expected; Lesser (1985) suggests the possibility of learned dorsiflexion of the toes in response to plantar stimulation of the foot. Hammond's (1948) case report of a psychopathic male patient who pressed on the side of his neck 
and self-induced an ipsilateral Babinski sign, increased patellar reflex and a dilated, fixed pupil is an intriguing example. Pseudo Todd's phenomena should also be envisaged (Hurwitz, 1988). The duration of an isolated convulsive pseudoseizure is variable, with a mean value above two minutes clearly suggestive of non epileptic tonic-clonic seizures (Gates et al., 1985).

A series of rapidly repeated convulsive pseudoseizures occurring without recovery of the baseline state between attacks and mimicking status epilepticus compel urgent medical attention and intervention. Pseudostatus is a real diagnostic problem and one often compounded by the development of potentially fatal iatrogenic complications. Some twenty-one cases are reported in the literature. Ventilatory assistance with or without cardiopulmonary resuscitation was required in eight of these (Table 1).

\section{EEG Monitoring}

Observation of the clinical manifestations alone, albeit useful to the experienced, has obvious diagnostic limitations (King et al., 1982). An EEG tracing recording one and if possible several habitual pseudoseizures is crucial evidence to gather at the crossroads of differential diagnosis.

In convulsive pseudoseizures, the tracings are likely to be obscured by muscle artifact. The absence of subtle electrical traces such as flattening of the EEG or an increase in spike and wave activity detected just prior to the attack as well as the absence of post-ictal flattening or slow waves become all the more important in excluding epileptic phenomenon (Trimble, 1986; Fenton, 1986; Desai et al., 1982). A well modulated alpha rhythm preserved during intervals between movement artifact, especially in a seemingly unresponsive patient, further supports the diagnosis of pseudoseizure.

TABle 1. Pseudostatus Epilepticus.

\begin{tabular}{lcc}
\hline Authors & No. of cases & $\begin{array}{c}\text { Ventilatory assistance } \\
\pm \text { C.P.R.* }\end{array}$ \\
\hline Liske (1964) & 1 & - \\
Toone (1979) & 3 & 2 \\
Ramani (1980) & 1 & - \\
Nicholl (1981) & 1 & - \\
Guberman (1982) & 1 & 1 \\
Cohen (1982) & 2 & unavailable \\
Krumholz (1983) & 7 & 2 \\
David (1985) & 1 & 1 \\
Fenton (1986) & 1 & unavailable \\
Cohen (1988) & 1 & 1 \\
Savard (1988) & 1 & - \\
Pankratz(1989) & 1 & 8 \\
$\quad$ Total & 21 & 1 \\
\hline
\end{tabular}

* C.P.R.: cardiopulmonary resuscitation 
To increase the likelihood that an actual pseudoseizure will occur while recording in the EEG laboratory, the use of suggestion has been recommended (Cohen and Suter, 1982; Luther et al., 1982; Krumholz and Niedermeyer, 1983).

It must be conceded that not all electrical activity of the brain, whether normal or epileptic, can be sampled at any given time: consideration must be given to the appropriateness of the EEG montage, the length of recording, presence or absence of videorecording, use of special leads and of activation methods. The absence of electrographic seizure activity never completely excludes the possibility of epilepsy. Seizures with bizarre phenomenology such as bicycling motions, fondling of the genitals, running or other semiautomatic motoric sequences can sometimes be ascribed to a frontal or to a parietal focus which may be silent on conventional EEG recording. Those two seizure types, both uncommon and ones with which many clinicians are unfamiliar with, can be misdiagnosed as pseudoseizures for some time before their true origin is revealed (Bancaud, 1989; Delgado-Escueta, 1989; Williamson, 1990).

\section{Neurohumoral Studies}

Serum prolactin studies provide an interesting approach to distinguish genuine epileptic convulsions from non epileptic ones. A five to thirty-fold rise of prolactin above baseline levels can be measured approximately 20 minutes after the majority of tonic clonic generalized seizures, after most complex partial seizures but only after some simple partial attacks. Such a rise is never present after convulsive pseudoseizures (Trimble, 1978, 1986). A gradual lessening of the expected prolactin rise occurs when serial epileptic seizures occur one after another: exhaustion of the central pituitary prolactin store may be the cause (Berkovic, 1984). One important implication is that the absence of a prolactin rise accompanying pseudostatus epilepticus is invalidated as the absence of such a rise is the rule in true status epilepticus (Tomson et al., 1989).

\section{Psychological Testing}

The Minnesota Multiphasic Personality Inventory (MMPI) is a well known psychological test which has been used in patients with pseudoseizures and in epileptic controls (Wilkus et al., 1984; Vanderzant et al., 1986; Heck et al., 1989).

While yielding important information about patients' psychopathological profiles, the MMPI does not permit differentiation between groups of matched patients with epileptic seizures and those with convulsive pseudoseizures (Wilkus and Dodrill, 1989).

\section{Psychiatric Etiology}

It must be realized that pseudoseizures can develop in patients suffering from various psychiatric diseases. 
The presence of pseudoseizures often heralds severe psychopathology, including major affective disorders and major personality disorders, both antisocial and borderline: patients with pseudoseizures often have a past history of suicide attempts and of psychiatric treatment (Stewart et al., 1982; Roy, 1979). Perhaps affective disorder underlies new onset pseudoseizures while adoption of the sick role and environmental reinforcing factors are more relevant in chronic pseudoseizures, long after depression has remitted (Roy, 1982).

Less frequently, pseudoseizures occur in the context of a somatization disorder or Briquet's hysteria (Steward et al., 1982).

Caution is warranted when pseudoseizures are thought to represent the sole manifestation of a conversion disorder. Primary symbolism of psychodynamic relevance and unconscious motivation are at best difficult to document in the psychoanalytic setting, and are treacherous to infer during a bedside psychiatric consultation (Lazare, 1981). Slater's famous warning expressed in 1965 that hysteria or conversion disorder is a "disguise for ignorance and a fertile source of clinical error" is still valid. His own view emerged from his survey of 85 patients followed for a mean duration of 10 years: twenty-two percent developed neurological illnesses and, among those still disabled, other psychiatric diagnoses emerged.

The issue of possible deceitful simulation by pseudoseizure patients is always present. Cases of malingering such as exemplified by the literary hero Felix Krull (Thomas Mann, 1955) are notoriously common in the military or forensic psychiatric setting. Cases of factitious disorder with pseudoseizures and even with convulsive pseudostatus epilepticus are scattered in the medical literature, and are likely under-reported (Savard et al., 1988; Savard and Andermann, 1990).

It has been claimed that, while 42 to $90 \%$ of pseudoseizure patients do not suffer from epilepsy, 8 to $20 \%$ of those admitted to comprehensive epilepsy centers because of medically intractable seizures have pseudoseizures alone or in combination with epilepsy (Ramani, 1986). In the epileptic patient otherwise predisposed to the development of pseudoseizures, the emergence of the pseudoconvulsive attacks appears to be facilitated by antiepileptic drug toxicity and treatment is facilitated by the prompt correction of blood levels (Niedermeyer et al., 1970; Reynolds, 1979).

The epileptic patient may develop depression which at times consists of an organic mood disorder adversely affected by anticonvulsant drug polytherapy, and which could manifest itself by pseudoseizures (Trimble, 1986). Other organic brain syndromes with personality or cognitive deterioration and mental retardation may also predispose epileptic patients to the development of pseudoseizures, whether organic alexithymia is present or not, in the context of endangered coping skills (Fenton, 1986; Lesser, 1985; Savard and Andermann, 1990). Such patients may at times embellish minor seizure symptomatology by lack of a better means of alerting their doctor to their uncontrolled epilepsy (Ramani, 1980). 


\section{Treatment}

The treatment of convulsive pseudoseizures thought to be of psychiatric etiology is naturally directed at the underlying psychopathology. A therapeutic alliance is first secured and a "face saving" approach is most important when psychic distress is disavowed by the patient. Education about the non-epileptic nature of the attacks with eventual review of videotaped episodes benefits most patients. In the introspective patient, a psychodynamically inspired approach helps to foster insight into the meaning of the pseudoseizure. Identification of triggering events, minimization of reinforcing factors and implementation of appropriate penalties by environmental manipulation are helpful, especially in a patient with low I.Q. or with little insight (Fenton, 1986; Lesser, 1985).

Timely use of psychopharmacological agents such as antidepressant medications is recommended, and an attempt to achieve carbamazepine monotherapy in the epileptic patient with pseudoseizures is rewarding at times (Blumer and Zielinski, 1988; Trimble, 1986).

\section{Prognosis}

Reliable figures concerning prognosis of pseudoseizures are not available. Improvement rates reported so far have varied between 30 and $80 \%$ (Lesser, 1985). Most patients identified in a neurological facility have a good prognosis while those who are diagnosed in a psychiatric institution do not seem to have as high a remission rate.

\section{Discussion}

Patients with pseudoseizures who do not suffer from coexistent epilepsy are at risk for unnecessary exposure to antiepileptic medication. Patients with epileptic seizures who develop pseudoseizures are at risk for an excessive dose and level of antiepileptic drugs and consequent toxicity. In that setting, pseudoseizures once initiated may not remit.

Psychiatric enquiry and treatment should be initiated early on, especially when dangerous features such as intentional injury are present.

Difficult diagnostic problems remain, especially in cases suspected of having parietal epilepsy whose bizarre symptomatology is often mistaken for conversion disorder (Bancaud, 1989; Lesser, 1983; Savard and Andermann, 1990). The same is true in cases of frontal lobe epilepsy (DelgadoEscueta, 1989; Williamson, 1990).

When diagnostic problems develop, thorough joint neurological and psychiatric follow-up is often necessary to prevent complications from untreated epilepsy misdiagnosed as pseudoseizures, or from unattended suicidal tendency underlying the pseudoseizures. Proof of the psychiatric causation can be difficult to demonstrate in cases where the psychopathology underlying the pseudoseizures is refractory to comprehensive psychiatric treatment. 
Most of what is known about convulsive pseudoseizures is now being applied to non convulsive pseudoseizures mimicking complex partial seizures (Remick and Wada, 1979; Andermann, personal communication). Pseudoseizures with predominant affective symptomatology may occur in a different subgroup of patient with distinguishing MMPI test profiles (Wilkus and Dodrill, 1989).

\section{References}

Andermann, F. (1990). Non epileptic paroxysmal neurological events. In "The Dilemma of Non-Epileptic (Pseudoepileptic) Seizures" (Eds A. J. Rowan and J. R. Gates). Demos Publications, New York, in press.

Bancaud, J. (1989). Localisation préopératoire du foyer du lobe pariétal et du lobe occipital. 50th Anniversary Symposium on presurgical evaluation and surgical therapy of focal epilepsy, Montreal, Canada.

Berkovic, S. (1984). Clinical and experimental aspects of complex partial seizures. Doctor of Medicine Thesis. University of Melbourne.

Blumer, D. and Zielinski, J. J. (1988). Pharmacologic treatment of psychiatric disorders associated with epilepsy. Fournal of Epilepsy, 1, 135-150.

Cohen, L. M., Bongar, B., Howard III, G. and White, R. (1988). Pseudostatus epilepticus. Psychosomatics, 29 (1), 116-118.

Cohen, R. J. and Suter, C. (1982). Hysterical seizures: suggestion as a provocative EEG test. Annals of Neurology, 11, 391-395.

David, A. S. and Bone, I. (1985). Hysterical paralysis following status epilepticus: case report and review of the concept. Fournal of Nervous and Mental Disease, 173 (7), 437-440.

Delgado-Escueta, A. (1989). Ictal manifestations of frontal lobe seizures. Neurobehavioral problems in epilepsy, an International Symposium, New Orleans, USA.

Desai, B. T., Porter, R. J. and Penry, J. K. (1982). Psychogenic seizures. Archives of Neurology, 39, 202-209.

Feldman, R. G. and Paul, N. L. (1976). Identity of emotional triggers in epilepsy. Fournal of Nervous and Mental Disorders, 162 (5), 345-353.

Fenton, G. W. (1986). Epilepsy and hysteria. British Journal of Psychiatry, 149, 28-37.

Gates, J. R., Ramani, V., Whalen, S., Loewenson, R. (1985). Ictal characteristics of pseudoseizures. Archives of Neurology, 42, 1183-1187.

Gowers, W. R. (1901). Hystero-epilepsy. In "Epilepsy and Other Chronic Convulsive Diseases: Their Symptoms, Causes and Treatment", 2nd edn. J. A. Churchill, London, pp. 164-192.

Green, S. A. (1977). A case of functional sleep seizures. Journal of Nervous and Mental Disease, $164(3), 223-227$.

Guberman, A. (1982). Psychogenic pseudoseizures in non-epileptic patients. Canadian fournal of Psychiatry, 27, 401-404.

Hammond, R. D. (1948). Simulated epilepsy: report of a case. Archives of Neurology and Psychiatry, 60, 327-328.

Heck, D. G., Brady, T. J., Barrash, J., Gates, J. R., Taylor, M. R. (1989). Nonepileptic events: a look at gender differences. Epilepsia, 30, 689.

Henry, J. and Woodruff, G. A. J. (1978). A diagnostic sign in states of apparent unconsciousness. Lancet, 2, 920-921.

Hurwitz, T. A. (1988). Narcosuggestion in chronic conversion symptoms using combined intravenous amobarbital and methylphenidate. Canadian Journal of Psychiatry, 33, $147-$ 152.

King, D. W., Gallagher, B. B., Murvin, A. J., Smith, D. B. et al. (1982). Pseudoseizures: diagnostic evaluation, Neurology (Ny), 32, 18-23.

Krumholz, A. and Neidermeyer, E. (1983). Psychogenic seizures: a clinical study with followup data. Neurology, 33, 498-502. 
Lazare, A. (1981). Current concepts in psychiatry: conversion symptoms. New England fournal of Medicine, 305, 745-748.

Lennox, W. G. (1960). "Epilepsy and Related Disorders". Vol. 1. Little, Brown and Company, Boston.

Lesser, R. P. (1985). Psychogenic seizures. In "Recent Advances in Epilepsy" (Eds T. Pedley and B. S. Meldrum) Vol. 2. Churchill Livingston, Edinburgh, pp. 273--296.

Lesser, R. P., Lueders, H., Conomy, J. P., Furlan, A. J. et al. (1983). Sensory seizures mimicking a psychogenic seizure. Neurology, 33 (6), 800-802.

Liske, E. and Forster, F. M. (1964). Pseudoseizures: a problem in diagnosis and management of epileptic patients. Neurology, 14, 41-49.

Luther, J. S., McNamara, J. O., Carwile, S., Miller, P. et al. (1982). Pseudoepileptic seizures: methods and video analysis to aid diagnosis. Annals of Neurology; 12, 458-462.

Mann, Thomas (1955). "Confessions of Felix Krull". Alfred A. Knopf, New York.

Nicholl, J. S. (1981). Pseudoseizures: a neuropsychiatric diagnostic dilemma. Psychosomatics, $22(5), 451-454$.

Niedermeyer, E., Blumer, D., Holscher, E. and Walker. B. A. (1970). Classical hysterical seizures facilitated by anticonvulsant toxicity. Psychiatria Clinica, 3, 71-84.

Pankratz, L. (1989). Historical note on pseudoseizures. Neurology, 39, 750.

Ramani, S. V., Quesney, L. F., Olson, D. and Gumnit, R. J. (1980). Diagnosis of hysterical seizures in epileptic patients. American Journal of Psychiatry, 137 (6), 705-709.

Ramani, V. (1986). Intensive monitoring of psychogenic seizures, aggression, and dyscontrol syndromes. In "Advances in Neurology" Vol. 46, Intensive Neurodiagnostic Monitoring (Ed. R. J. Gumnit). Raven Press, New York, pp. 203-217.

Remick, R. A. and Wada, J. A. (1979). Complex partial and pseudo seizure disorders. American Fournal of Psychiatry, 136 (3), 320-323.

Reynolds, E. H. (1989a) Phenytoin toxicity. In "Antiepileptic Drugs" (Eds R. H. Levy, R. Mattson, B. Meldrum, J. K. Penry and F. E. Dreifuss) 3rd edn. Raven Press, New York, p. 243.

Reynolds, E. H. (1989b) Carbamazepine toxicity. In "Antiepileptic Drugs" (Eds R. H. Levy, R. Mattson, B. Meldrum, J. K. Penry and F. E. Dreifuss) 3rd edn. Raven Press, New York, p. 556.

Rosenberg, M. M. L. (1986). Geotropic eye movements and pseudoseizures. Archives of Neurology, 43, 544.

Roy, A. (1979). Hysterical seizures. Archives of Neurology, 36, 447.

Roy, A. (1982). Hysterical neurosis. In "Hysteria" (Ed. A. Roy). John Wiley, New York, pp. 89-98.

Savard, G., Andermann, F., Teitelbaum, J. and Lehmann, H. (1988). Epileptic Munchausen's syndrome: a form of pseudoseizures distinct from hysteria and malingering. Neurology, 38 (10), 1628-1629.

Savard, G., Andermann, F. (1990). Pseudoseizures: interface between neurological and psychiatric events. In "The Dilemma of Non-Epileptic (Pseudoepileptic) Seizures" (Eds A. J. Rowan and J. R. Gates). Demos Publications, New York, in press.

Slater, E. (1965). Diagnosis of hysteria. British Medical Fournal, 1, 1395-1399.

Stewart, R. S., Lovitt, R. and Stewart, R. M. (1982). Are hysterical seizures more than hysteria? American Journal of Psychiatry, 139, 926-928.

Tomson, T., Lindbom, U., Nilsson, B. Y., Svanborg, E., Andersson, D. E. H. (1989). Serum prolactin during status epilepticus. Fournal of Neurology, Neurosurgery, and Psychiatry, 52, 1435-1437.

Toone, B. K. and Roberts, J. (1979). Status epilepticus: an uncommon hysterical conversion syndrome. Journal of Nervous and Mental Disease, 167 (9), 548-552.

Trimble, M. R. (1978). Serum prolactin in epilepsy and hysteria. British Medical Journal, 2, 1682-1978.

Trimble, M. R. (1986). Pseudoseizures. In "Neurologic Clinics" (Eds R. J. Porter and N. H. Theodore) Vol. 4. W. B. Saunders, Philadelphia, pp. 531-548.

Vanderzant, C. W., Giordani, B., Berent, S., Dreifuss, F. E., Sackellares, J. C. (1986). Personality of patients with pseudoseizures. Neurology, 36, 664-668. 
Wilkus, R. J. and Dodrill, C. B. (1989). Factors affecting the outcome of MMPI and neuropsychological assessment of psychogenic and epileptic seizure patients. Epilepsia, 30, 339-347.

Wilkus, R. J., Dodrill, C. B. and Thompson, P. M. (1984). Intensive MMPI monitoring and psychological studies of patients with pseudoepileptic seizures. Epilepsia, 25, 100-107.

Williamson, P. D. (1990). Frontal seizures vs non epileptic seizures. In "The Dilemma of Non-Epileptic (Pseudoepileptic) Seizures" (Eds A. J. Rowan and J. R. Gates). Demos Publications, New York, in press. 




The Scientific World Journal
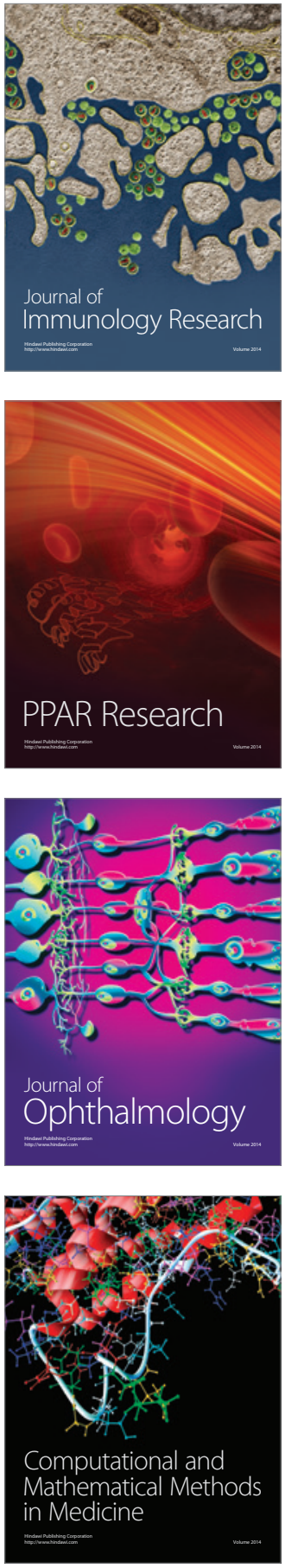

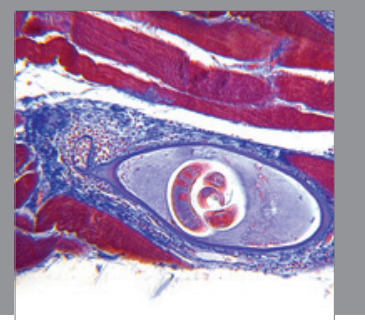

Gastroenterology

Research and Practice


\section{Hindawi}

Submit your manuscripts at

http://www.hindawi.com


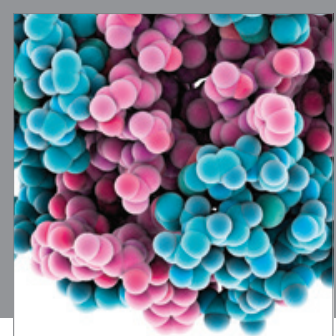

Journal of
Diabetes Research

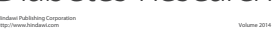

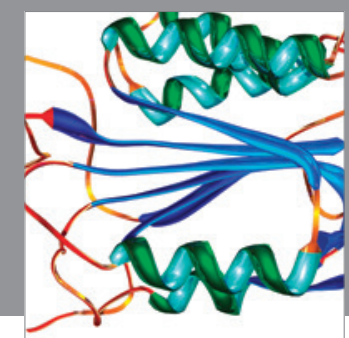

Disease Markers
